NOUVELLES ETUDES CONCERNANT L'ALIMENTARITE : Evaluation de la sécurité des aliments issus d'organismes génétiquement modifiés : acquis et incertitudes

NEW RESEARCH ON FOOD SAFETY: An evaluation of the safety of foods produced from genetically modified crops: what is certain and what isn't

Oléagineux, Corps Gras, Lipides. Volume 7, Numéro 4, 310-3, Juillet - Août 2000, Dossier : "OGM: expertise et décision publique"

Auteur(s) : Pierre BESANÇON, Université de Montpellier, Laboratoire de génie biologique et sciences des aliments, place E.-Bataillon, 34095 Montpellier Cedex 05.

Résumé : Une très grande variété de produits alimentaires issus d'organismes génétiquement modifiés sont proposés - ou susceptibles de l'être - à la consommation humaine, puisque les techniques du génie génétique sont applicables aussi bien aux micro-organismes qu'aux végétaux et aux animaux. La plupart d'entre eux sont considérés comme de nouveaux aliments ou de nouveaux ingrédients alimentaires et relèvent du règlement européen EC 258-97 [1]. À ce titre, l'évaluation de leur innocuité s'impose.

Summary: Numerous food products may be derived from GMO. A risk assessment is required in each case, involving four steps: - an analysis of the genetic construction, - a biochemical, functional and toxicological characterisation of the new expressed proteins, - a determination of the nutritional value of the new food, - an evaluation of unintended effects. Subchronic-tests are preferred to acute toxicity assays. Controlling the degradability of new proteins by digestive simulation tests and mutagenicity tests may be discussed. Lastly it is also recommended to perform tolerance studies by feeding experimental animals with the final new products.

Keywords: GMO foods, risk assessment, toxicity, nutritional value.

\title{
ARTICLE
}

L'analyse du risque distingue classiquement les risques liés à la dissémination des transgènes, en particulier de gènes de résistance à des antibiotiques, qui ne seront pas évoqués ici et les risques éventuels liés à la consommation animale ou humaine. La très grande diversité des produits qui existent déjà ou qui pourraient être proposés dans un avenir plus ou moins proche fait que les niveaux de risque en alimentation humaine peuvent être très différents selon que l'on considère :

- des plantes génétiquement modifiées dont les seules parties comestibles sont directement consommables par l'homme (fruits, légumes, chicorée, maïs doux...) ;

- des plantes génétiquement modifiées destinées à une transformation industrielle et à l'élaboration de produits dits intermédiaires, eux-mêmes utilisables comme ingrédients alimentaires (amidons de 
maïs, de blé, sirops de glucose ou de fructose issus de ces amidons, amidon modifié de pomme de terre, lécithines de soja...);

- des plantes génétiquement modifiées destinées à l'alimentation animale sous la forme de fourrages ou de farines (maïs, soja), destinés à des animaux d'élevage dont seuls les produits (lait, viande, œufs) seront consommés en alimentation humaine ;

- des animaux ayant fait l'objet de nouvelles technologies de la reproduction dites de $3^{\mathrm{e}}$ ou de $4^{\mathrm{e}}$ génération, telles que la fécondation in vitro, le clonage par transfert de noyaux, la transgenèse [2] et dont les produits seraient directement consommés par l'homme ;

- des micro-organismes génétiquement modifiés utilisables directement soit comme probiotiques, soit comme ferments dans des produits fermentés aussi traditionnels que des fromages ou du vin ;

- des micro-organismes génétiquement modifiés utilisés comme producteurs d'auxiliaires technologiques, tels que des enzymes elles-mêmes mises en œuvre dans l'industrie agro-alimentaire pour améliorer ou modifier des produits ou des procédés industriels [3].

L'évaluation de la sécurité sanitaire de tels produits doit donc non seulement tenir compte de la nature de la transformation génétique et de l'espèce qui en fait l'objet, mais aussi du stade de la chaîne alimentaire auquel on se place ainsi que des différentes transformations. En particulier, le transgène et ses produits d'expression sont présents dans les parties comestibles des plantes et animaux génétiquement modifiés, sauf exception ; en revanche, le transgène devrait être théoriquement absent, sauf à l'état de traces, dans les ingrédients issus de produits intermédiaires et a fortiori dans les produits traités par des enzymes recombinantes.

\section{Lignes directrices}

L'évaluation de l'innocuité de ces nouveaux aliments repose donc sur un examen au cas par cas. II n'en demeure pas moins indispensable de coordonner et d'harmoniser les procédures d'évaluation. Suite aux travaux de nombreux groupes [4-6], les principes présidant à l'évaluation de la sécurité d'emploi des nouveaux aliments ont été définis et développés.

Un cadre plus formel d'évaluation a été proposé sous la forme d'arbres de décision et de recommandations de nature scientifique par le Comité scientifique de l'alimentation humaine [7]. II a paru nécessaire d'aller plus loin dans ces recommandations et d'apporter un cadre méthodologique par l'établissement de lignes directrices. C'est, entre autres, ce qu'a proposé le Conseil supérieur d'hygiène publique de France qui a adopté, par un avis du 12 janvier 1999, des lignes directrices concernant les plantes génétiquement modifiées et les produits alimentaires qui en sont issus [8]. II y a généralement un consensus pour porter l'analyse aux différents niveaux suivants :

- analyse de la construction génétique ;

- analyse et caractérisation physico-chimique, fonctionnelle et toxicologique des produits d'expression ;

- valeur nutritionnelle des produits et équivalence en substance ; 
- recherche d'effets non intentionnels et inattendus.

Une analyse critique portant sur l'évaluation de l'insécurité des nouveaux aliments a récemment été proposée par Wal et Pascal [9].

Enfin, il faut admettre que beaucoup reste à faire pour établir des lignes directrices adaptées aux produits issus d'animaux transgéniques ainsi qu'aux micro-organismes génétiquement modifiés destinés à être consommés directement comme ferments ou comme probiotiques.

En revanche, s'il n'existe pas encore de réglementation communautaire concernant les enzymes utilisées comme auxiliaires technologiques, des lignes directrices ont été établies dès 1991 par le Comité scientifique de l'alimentation humaine, pour l'évaluation des préparations enzymatiques, y compris celles issues de micro-organismes génétiquement modifiés. Ces lignes directrices portent sur

- l'organisme producteur, la démonstration de sa non-pathogénicité, la description des constructions génétiques (organisme donneur, hôte, nature du transgène...) ;

- la préparation enzymatique (critères de pureté, innocuité, activités enzymatiques secondaires...) ;

- les effets de l'enzyme sur les produits alimentaires traités.

Les procédures appliquées en France et les critères de pureté requis ont été décrits par ailleurs [10]. Le Conseil supérieur d'hygiène publique de France a également émis un avis selon lequel on doit démontrer l'absence d'ADN recombinant dans les préparations enzymatiques issues d'OGM.

Qu'elles s'appliquent aux plantes transgéniques ou aux enzymes, ces lignes directrices ont bien évidemment leurs limites. Certaines des informations requises ne posent pas de problème majeur, parce qu'elles reposent sur des méthodes sûres et validées : il s'agit en particulier de l'analyse de la construction génétique (séquences transférées, nombre d'insertions, stabilité, possibilités de transferts, informations d'ordre analytique pour assurer la traçabilité), de la caractérisation des produits d'expression aux niveaux biochimique et fonctionnel. En revanche, la caractérisation toxicologique pose généralement plus de problèmes, parce qu'il s'agit précisément de protéines.

\section{Évaluation toxicologique des protéines exprimées}

La détermination des niveaux d'expression des protéines nouvelles, exprimées dans les différents tissus et organes de la plante, ne devrait pas poser de problème particulier avec les techniques analytiques actuelles, notamment immunochimiques.

L'évaluation toxicologique pourrait dans un premier temps tenir compte des fonctions exercées par la nouvelle protéine. Ainsi, la protéine cry IA (b) de Bacillus thuringiensis, introduite dans le maïs pour lui conférer la résistance à la pyrale, n'est considérée comme toxique que chez certains lépidoptères : cette DELTA-endotoxine est agoniste d'un récepteur présent dans l'intestin moyen de l'insecte. Ce récepteur est absent chez tous les autres animaux et en particulier chez les mammifères, donc chez l'homme chez qui la DELTA-endotoxine n'interagit pas avec la muqueuse intestinale. De même, une enzyme recombinante telle que la chymosine n'a pas de fonction 
particulière, au niveau physiologique, autre que la coagulation des caséines, par hydrolyse de la kappa-caséine.

En revanche, la plupart des enzymes qui auraient un effet physiologique (protéases, transglutaminase) doivent être inactivées dans le produit alimentaire ou éliminées. Ces données très spécifiques n'excluent pourtant pas des essais de toxicologie générale.

Les protéines PAT (résistance au glufosinate) et EPSPS (résistance au glyphosate) sont les deux principales protéines impliquées dans la résistance aux herbicides : les données actuellement disponibles ou publiées font état des tests de toxicité aiguë ( $D L 50$ déterminée dans des conditions expérimentales simplifiées) et de tests de dégradabilité enzymatique in vitro.

Il est généralement admis que des tests de toxicité subchronique, par administration quotidienne réitérée sur une durée de 90 jours ou à la rigueur de 28 jours, sont les plus adéquats pour des substances qui sont censées être présentes, même à des doses très faibles, dans des produits alimentaires destinés à être consommés régulièrement. En revanche, des tests de toxicité chronique sur 2 ans poseraient de difficiles problèmes de réalisation et n'apporteraient pas d'informations supplémentaires significatives. On préconise donc un test de 90 jours sur des rats pris au départ en période de pleine croissance.

La principale objection faite à l'encontre des résultats ainsi obtenus tient au fait qu'il est difficile d'obtenir des protéines en quantité suffisante pour réaliser de tels tests : soit on extrait la protéine de la plante qui l'exprime et on risque de la dénaturer au cours de l'extraction, soit on fait exprimer cette protéine dans une bactérie, pour en obtenir des quantités significatives. Dans ce dernier cas, on court le risque d'obtenir une protéine de conformation légèrement différente, puisque les régulations post-traductionnelles sont différentes (pas de glycosylation). Dans quelques cas limités, il a été possible de recourir à cette stratégie lorsque la protéine à tester n'est pas glycosylée.

Les tests de dégradabilité in vitro sur des systèmes modèles simulant la digestion gastrique et intestinale posent problème dans la mesure où ils sont pratiqués dans des conditions expérimentales relativement simplifiées par rapport à la réalité physiologique. Ces tests consistent à suivre la dégradation des protéines d'abord dans un milieu à $\mathrm{pH} 2$ en présence de pepsine puis à $\mathrm{pH} 7$ en présence d'extrait pancréatique. II arrive que ces tests soient réalisés séparément et non de façon séquentielle, ce qui n'a plus de signification physiologique. Ces essais ne tiennent pas compte des effets de matrice de l'aliment dans les contenus digestifs (pouvoir tampon), des cinétiques de sécrétion des enzymes digestives, des cinétiques d'hydrolyse, des produits intermédiaires d'hydrolyse pouvant résister longtemps à l'hydrolyse dans l'intestin.

Les tests de génotoxicité sont-ils pertinents ?

Pour les préparations enzymatiques, les lignes directrices européennes préconisent deux tests, l'un de mutagénicité (par exemple un essai de mutagenèse inverse), l'autre de clastogénicité. Ces tests sont parfaitement appropriés pour des substances autres que des protéines; dans le cas des préparations enzymatiques, ces tests sont pertinents pour détecter le pouvoir génotoxique d'impuretés potentiellement présentes. L'examen de dizaines de dossiers montre que les préparations enzymatiques ne révèlent jamais de potentiel génotoxique dans la mesure où les pratiques de sélection des souches, d'autoclonage et d'amplification de gènes conduisent à des 
préparations de plus en plus pures et de plus en plus actives, avec des facteurs de sécurité de plus en plus élevés, ce qui est encourageant !

L'évaluation du potentiel allergénique a été largement décrite et discutée par ailleurs $[9,11,12]$; il y a encore sujet à débat. Cependant, il y a un consensus pour admettre que les techniques du génie génétique ne sont pas de nature à conférer un potentiel allergénique aux produits obtenus, sauf si l'on introduit un gène codant pour une protéine considérée comme allergénique. Ce fut le cas de la protéine $2 \mathrm{~S}$ de la noix du Brésil, qui a été abandonnée. Les données actuellement disponibles font état, d'une part, de tests de dégradabilité en système digestif simulée et, d'autre part, de la recherche d'homologies de séquences portant sur 8 acides aminés consécutifs, par comparaison avec des protéines connues pour leur potentiel allergénique, ce qui exclut $a$ priori les épitopes à caractère conformationnel.

Un autre problème émergent est lié aux techniques permettant de modifier spécifiquement un ou plusieurs acides aminés dans la séquence d'une protéine. L'argument consistant à affirmer que la protéine modifiée présente un degré d'homologie très fort par rapport à la protéine de référence n'est pas suffisant ni recevable sur le plan sécuritaire. Dans ce cas, il faut considérer la protéine modifiée comme une nouvelle protéine, à évaluer sur le plan toxicologique. Ceci concerne les enzymes modifiées pour améliorer leur stabilité ou leur activité dans des conditions de milieu inhabituelles. C'est aussi le cas de protéines modifiées sur quelques acides aminés et dont le gène a été transféré à une plante. La même remarque peut être faite pour des protéines de fusion, même si l'on considère que les deux protéines constitutives ne sont pas toxiques.

\section{Valeur nutritionnelle, équivalence en substance, effets non intentionnels}

Le principe d'équivalence en substance a été longuement débattu et peut s'appliquer fort bien à des nouveaux aliments issus de nouvelles technologies qui n'impliquent pas de modifications du génome.

L'équivalence en substance peut théoriquement être établie à partir de l'analyse de l'ensemble des macro et micro-nutriments, des facteurs anti-nutritionnels et/ou toxiques, connus dans la famille botanique considérée.

L'interprétation des données, notamment sur le plan statistique est toujours difficile : variabilité intraspécifique, effets environnementaux, conditions de culture. Dans le cas des OGM, on devrait admettre qu'il n'est pas possible d'appliquer le principe d'équivalence en substance, ne serait-ce que par la possibilité d'effets pléitropes, traduisant des modifications du génome (altération d'autres gènes, stimulés ou réprimés) ou des modifications de régulation post-traductionnelle (glycosylation, phosphosylation), mais dont les conséquences sont difficiles à déterminer analytiquement. Les techniques d'analyses par empreintes génétiques (génome) ou métaboliques (protéome) sont donc à développer.

Ceci étant dit, la détermination de la valeur nutritionnelle d'une plante transgénique peut se faire, le cas échéant, au niveau de la plante entière et, dans le plus grand nombre de cas, sur les différentes familles de produits alimentaires qui en sont issus après transformation technologique.

II n'y a pas d'autre solution, dans un premier temps, que d'en déterminer analytiquement la composition en nutriments et en substances antinutritionnelles ou à activité biologique. Cependant, 
la valeur nutritionnelle est le reflet non seulement d'une table de composition mais aussi, notamment, de la biodisponibilité des nutriments.

C'est la raison pour laquelle l'idée prévaut actuellement de recommander la réalisation d'essais in vitro permettant de tester la tolérance du nouvel aliment dans des conditions nutritionnelles correctes : respect d'un bon équilibre nutritionnel, couverture des besoins nutritionnels. Ce type d'expérimentation ne constitue ni un essai nutritionnel, ni un essai toxicologique puisqu'il ne permet pas d'établir de relation dose-effet. Un test de tolérance devrait néanmoins permettre de donner quelques indications sur le plan nutritionnel (digestibilité, croissance) mais devrait aussi permettre, s'il est réalisé sur une période suffisamment longue incluant chez un jeune animal les phases de maturation pubertaire et de croissance intense, de révéler toute anomalie non décelable par ailleurs à partir de la simple démarche analytique. Le choix de l'espèce animale peut se discuter. Un bon essai réalisé sur une espèce d'intérêt zootechnique, dans des conditions respectant de bonnes pratiques d'expérimentation, est tout aussi acceptable qu'un test sur animaux de laboratoire : s'il est pratiqué sur le rat, une durée de 90 jours paraît souhaitable.

\section{CONCLUSION}

La tendance est actuellement au renforcement des études visant à établir l'innocuité de toute nouvelle protéine étrangère introduite par la voie génétique, au moyen des tests de toxicité subchronique et non par des tests de toxicité aiguë.

L'autre effort de démonstration de l'innocuité doit porter sur les produits alimentaires issus de I'OGM. Il est évident que la démonstration préalable de l'absence de toxicité de la protéine nouvelle exprimée ne dispense en rien de la réalisation de tests démontrant la bonne valeur nutritionnelle et la tolérance du produit alimentaire final.

L'application stricte des lignes directrices actuellement proposées nous interpelle aussi $a$ contrario sur l'exacte connaissance que nous devrions avoir des aliments considérés comme traditionnels. Nombre de questions posées (allergénicité, cinétiques d'hydrolyse digestive, effets biologiques autres que nutritionnels des constituants de nos aliments) ne sont pas spécifiques aux produits issus du génie génétique.

\section{REFERENCES}

1. Règlement européen sur les nouveaux aliments et nouveaux ingrédients alimentaires EC 258-97 (1997). Journal Officiel des Communautés européennes, 14 février.

2. THIBIER M, Coord. (1999). Colloque scientifique 29 septembre 1999. Biotechnologies de la reproduction animale et sécurité sanitaire des aliments. Maisons-Alfort : AFSSA Ed, 1999 : 118.

3. LARRETA-GARDE V, Coord. (1997). Enzymes en agro-alimentaire. Paris : Lavoisier Tec \& Doc Ed: 380. 
4. WHO (1995). Application of the principle of substantial equivalence to the safety evaluation of food components from plant derived by modern biotechnology. Genève : WHO, Food safety unit : 63.

5. FAO-WHO. Biotechnology and food safety. Joint FAO/WHO Expert Committee, WHO.

6. Organisation pour la coopération économique et le développement (1996). Food safety evaluation. Paris : OCDE Publication.

7. Journal Officiel des Communautés européennes, 16 septembre 1997, L.253, Vol. 40.

8. BESANÇON P (1999). Évaluation de la sécurité des nouveaux aliments. OCL, $6:$ 286-9.

9. WAL JM, PASCAL G (1999). Nouveaux aliments, nouveaux risques. Analyse et évaluation des risques liés à la consommation de nouveaux aliments. Médecine et Nutrition, 35 : 165-84.

10. BESANÇON P (1997). Les principes de la régulation sur l'utilisation des enzymes dans les industries alimentaires. In : LARRETA-GARDE V, Coord. Les enzymes. Paris : Tec \& Doc Lavoisier Ed : 13-25.

11. METCALFE DD, ASTWOOD JD, TOWNSEND R, SAMPSON HA, TAYLOR SL, FUCHS RL (1996). Assessment of the allergenic potential of foods derived from genetically engineered crop plants. Crit Rev Food Sci Nutr, 36 : S165-86.

12. CHESSON A, JAMES P (2000). Les aliments avec OGM sont-ils sans danger ? La Recherche, 327 : 27-35. 